\title{
Horizons/Théâtre
}

Revue d'études théâtrales

10-11 | 2017

Genre et arts vivants

\section{Trois œuvres dérivées, trois portraits plasticiens avec Denis D'Arcangelo et son double théâtral, Mme Raymonde}

\section{Lorraine Alexandre}

\section{(2) OpenEdition \\ 1 Journals}

Édition électronique

URL : http://journals.openedition.org/ht/511

DOI : 10.4000/ht.511

ISSN : 2678-5420

Éditeur

Presses universitaires de Bordeaux

\section{Édition imprimée}

Date de publication : 1 juillet 2017

Pagination : 96-112

ISSN : 2261-4591

\section{Référence électronique}

Lorraine Alexandre, «Trois œuvres dérivées, trois portraits plasticiens avec Denis D'Arcangelo et son double théâtral, Mme Raymonde », Horizons/Théâtre [En ligne], 10-11 | 2017, mis en ligne le 01 juillet 2018, consulté le 21 juillet 2019. URL : http://journals.openedition.org/ht/511 ; DOI : 10.4000/ht.511

\section{(9) $(\mathbb{\Theta \Theta}$}

La revue Horizons/Théâtre est mise à disposition selon les termes de la Licence Creative Commons Attribution - Pas d'Utilisation Commerciale - Pas de Modification 4.0 International. 


\section{LORRAINe ALEXANDRE}

Lorraine Alexandre est docteure en art et sciences de l'art. Rattachée à Acte (ancien CERAP) à Paris 1 Panthéon-Sorbonne, elle soutient en 2008 une thèse intitulée : Persona - La pratique des apparences comme acte de création où elle analyse les enjeux des mises en scène et des réappropriations formelles du corps dans l'ensemble des arts. Elle est l'auteure du livre Les Enjeux du portrait en art - Étude des rapports modèle, portraitiste, spectateur (L'Harmattan, série image de la théorie/théorie de l'image, 2011). Elle publie régulièrement notamment sur les études de genre avec, par exemple: "Mylène Farmer et Brigitte Bardot chez les travestis de genre : enjeux d'une référentialité », dans Barbara Lebrun (dir.), Chanson et performance. Mise en scène du corps dans la chanson française et francophone, Paris, L'Harmattan, 2012 ; "La fabrique du récit genré et sa re-signification : approche artistique » dans Patrick Farges, Cécile Chamayou-Khun et Perin Emel Yavuz (dir.), Le Lieu du genre - La narration comme espace performatif du genre, Paris, Presses de la Sorbonne nouvelle, 2011.

Mail : lorraine-a@hotmail.fr

Résumé: Personnage créé en 1988 par Denis D'Arcangelo, Philippe Bilheur et Arletty, Mme Raymonde fut d'abord héroïne de théâtre de rue. Elle propose depuis 2001 des tours de chants articulant théâtre, chanson et référence au cinéma. Les séries photographiques en trois parties de Lorraine Alexandre sont ici analysées: La galerie de portraits éclectiques évoque la place des femmes au théâtre et l'ambiguïté de la perception du personnage de Mme Raymonde par le public ; Denis/ Raymonde représente la métamorphose du comédien dans la peau de son clown et montre les coulisses d'une création théâ-

Abstract: Character created in 1988 by Denis D'Arcangelo, Philippe Bilheur and Arletty, Mrs Raymonde was at first heroin of street theater. She proposes since 2001 song recitals articulating theater, song and reference to the cinema. Lorraine Alexandre's photographic series in three parts are analysed here: "The Gallery of Eclectic Portraits" evokes women's place in theatre and the ambiguity of the perception of the character of Mrs Raymonde by the audience; "Denis/ Raymonde" represents the metamorphosis of the actor in his clown and shows the backstage of a theatrical creation and the stakes raised the inversion of gender; "Le portrait trale et les enjeux qu'elle soulève par l'inversion de genre; Le portrait affleurant de Mme Raymonde marque le processus de projection identification cher à Morin et les jeux $d^{\prime}$ intertextualité où les arts se rencontrent et s'associent. Ces photographies performatives, images construites et mises en scène, enrichissent la démarche du personnage de Denis D'Arcangelo, Mme Raymonde, qui devient sujet d'une création plasticienne.

Mots-CLÉs : travesti, photographie, projection identification, identité, féminisme

affleurant de Mme Raymonde" emphasizes the process of projection identification (Morin) and the intertextuality of arts thatmeet and join. These performative photos, constructed and staged images, enrich the approach of Denis D'Arcangelo's character, Mrs Raymonde, who becomes subject of an artwork.

KEYwORDS: travesty, photography, projection identification, identity, feminism 


\section{Trois œuvres dérivées, trois portraits plasticiens avec Denis D'Arcangelo et son double théâtral, Mme Raymonde}

Docteur en art et sciences de l'art, mention arts plastiques, je suis artiste plasticienne chercheuse et développe une pensée théorique à partir, et au regard, de ma pratique artistique. Mon travail interroge les modes de mises en scène et de réappropriations formelles du corps dans l'ensemble des arts m’amenant à faire régulièrement poser des artistes du spectacle (comédiens, chanteurs, Drag Queens, transformistes, acrobates et danseurs). J'ai notamment étudié, à travers une douzaine de séries photographiques et différentes publications, les enjeux des pratiques Drag en collaborant avec une dizaine de transformistes et Drag Queens. J'aimerais ici faire évoluer ces analyses déjà existantes vers un exemple théâtral avec le personnage de Madame Raymonde, interprétée par l'acteur chanteur Denis D’Arcangelo en hommage au personnage de la prostituée homonyme d'Arletty dans Hôtel du Nord de Marcel Carné (1938). Créée en 1988 par Denis D’Arcangelo et Philippe Bilheur avec la complicité d'Arletty, Mme Raymonde fut d'abord héroïne de théâtre de rue pendant douze ans. Elle propose depuis 2001 des tours de chants articulant théâtre, chanson et référence au cinéma. Denis D’Arcangelo y attire l'attention sur des figures de femmes fortes, volontaires et gouailleuses comme Arletty ou Brigitte Fontaine et protège de l'oubli des chanteuses peu connues du grand public actuel comme Gaby Montbreuse ${ }^{1}$.

Ma rencontre en 2011 avec Denis D’Arcangelo a représenté une évolution dans ma pratique d'artiste et dans la pensée qui l'accompagne. Nombreux sont les spectateurs et les journalistes qui le confondent avec les Drag Queens et les transformistes. Cependant, Denis D’Arcangelo n'est pas un travesti de genre qui intègre cette démarche à sa vie privée. Il est comédien et son personnage Mme Raymonde est une femme. Sa fidélité à ce personnage qu'il revendique aujourd' hui comme son double, ainsi que la dominance des personnages féminins dans sa carrière, fait sens au regard de sa démarche et de son parcours. Je me permettrai ici de présenter notre collaboration artistique sous forme de trois séries photographiques, et donc de trois parties, qui for- 
ment ma vision de portraitiste de Denis D’Arcangelo et de son double et qui deviennent ainsi trois révélateurs de la démarche artistique de mon modèle.

La galerie de portraits éclectiques, la plus simple en apparence, permet de revenir sur la place des femmes au théâtre et l'ambiguité de la perception du personnage de Mme Raymonde par le public. Denis/Raymonde, quatre photos en pied qui montrent le passage de Denis en Raymonde, représente la métamorphose du comédien dans la peau de son clown, comme il aime à l'appeler, et nous permettra de voir les coulisses d'une création théâtrale et les enjeux qu'elle soulève par l'inversion de genre. Le portrait affleurant de Mme Raymonde marque pour sa part le processus de projection identification cher à Edgard Morin et les jeux d'intertextualité entre différentes pratiques et références artistiques.

Il est important de préciser ici la nature d'œuvre dérivée de ma création. Je ne suis pas photographe mais plasticienne ; les photographies que je crée ne documentent pas mon sujet, mais font elles-mêmes œuvres. En termes juridiques, l'œuvre dérivée est une création originale ayant pour particularité d'avoir pour sujet l'œuvre préexistante d'un autre artiste.

Je ne portraiture presque jamais les individus comme tels. En tant que portraitiste, je suis la conviction d'Oscar Wilde lorsqu'il estime que « la forme objective est, en définitive, la plus subjective. L' homme cesse d'être lui-même lorsqu'il parle pour son propre compte. Donnez-lui un masque et il vous dira la vérité ${ }^{2} \gg$.

\section{Mme Raymonde, galerie de portraits éclectiques}

Mme Raymonde, galerie de portraits éclectiques est une série de vingt et une photographies argentiques en noir et blanc, format $6 \times 6$ (carré) représentant des portraits tête/épaules du personnage de Mme Raymonde. Certaines photos sont posées, d'autres prises sur le vif... Il est presque impossible pour le spectateur de les distinguer. J'ai créé cette série pour jouer avec le polymorphisme de ce comédien qui cumule et emmêle volontiers les émotions tant son visage est expressif. Mais ces photos tendent essentiellement à produire un effet d'empathie chez le spectateur.

Les tours de chant que Denis D’Arcangelo donne sous le masque de Mme Raymonde jouent avec l'ambivalence d'un personnage théâtral, mais suffisamment réaliste pour provoquer une proximité avec le public par un phénomène d'identification. La nature fictive est soulignée par le fait que cette femme est interprétée par un homme. L'inversion de genre provoque 
un décalage formel qui se doit de distinguer l'interprète de son personnage. Le rappel à la fiction installe alors le contexte théâtral. Mais le spectateur ne l'accepte pas toujours. Il n'est, par exemple, pas rare qu'il confonde cet acteur avec un transformiste de cabaret ${ }^{3}$. Au-delà d'un problème de méconnaissance du public, cette confusion pointe les enjeux du travestissement de genre troublant pour le spectateur. Très épurés, les tours de chant de Mme Raymonde se concentrent sur l'essentiel. Sans décors (à l'exception d'un siège et d'une petite table pour poser une bouteille de vin et deux verres), ils valorisent le personnage. Un unique musicien est aussi présent, l'accordéoniste Sébastien Mesnil dit le Zèbre. De façon générale, ce personnage, riche de ses ambiguïtés, dessine très vite des liens avec son public.

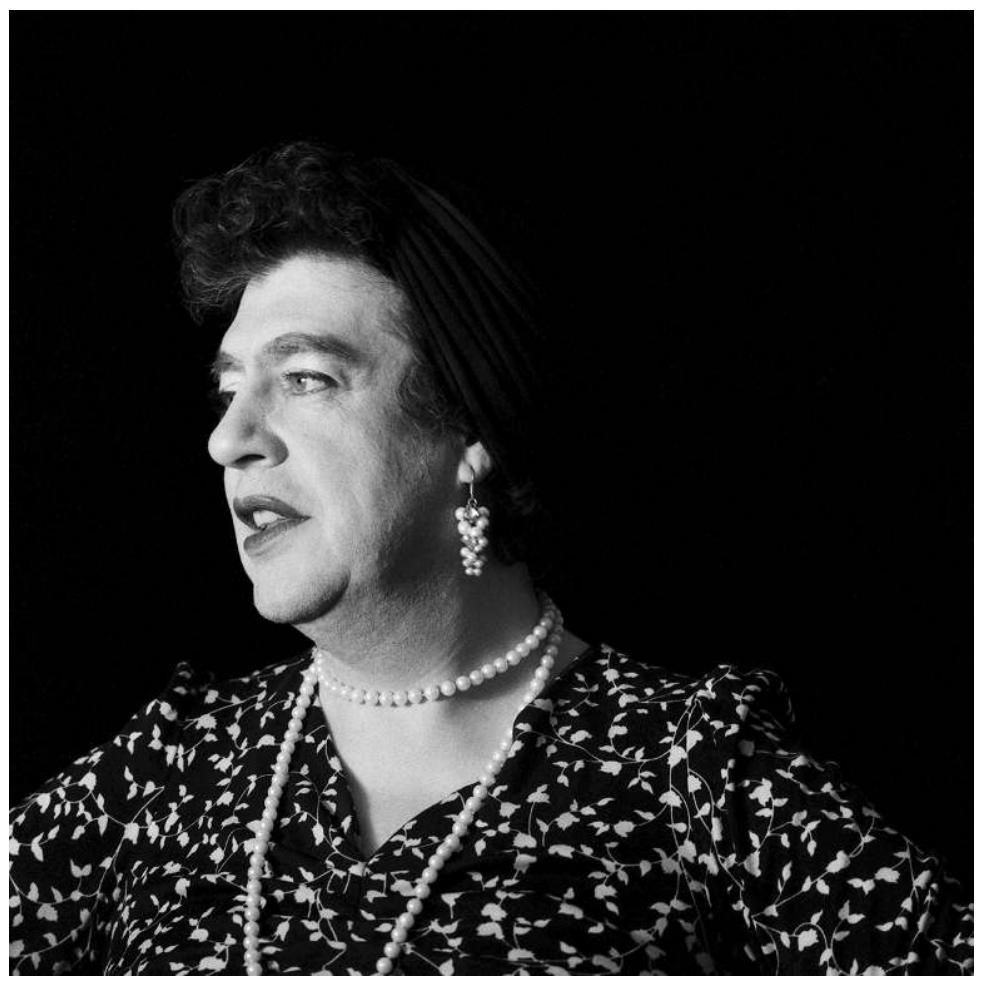




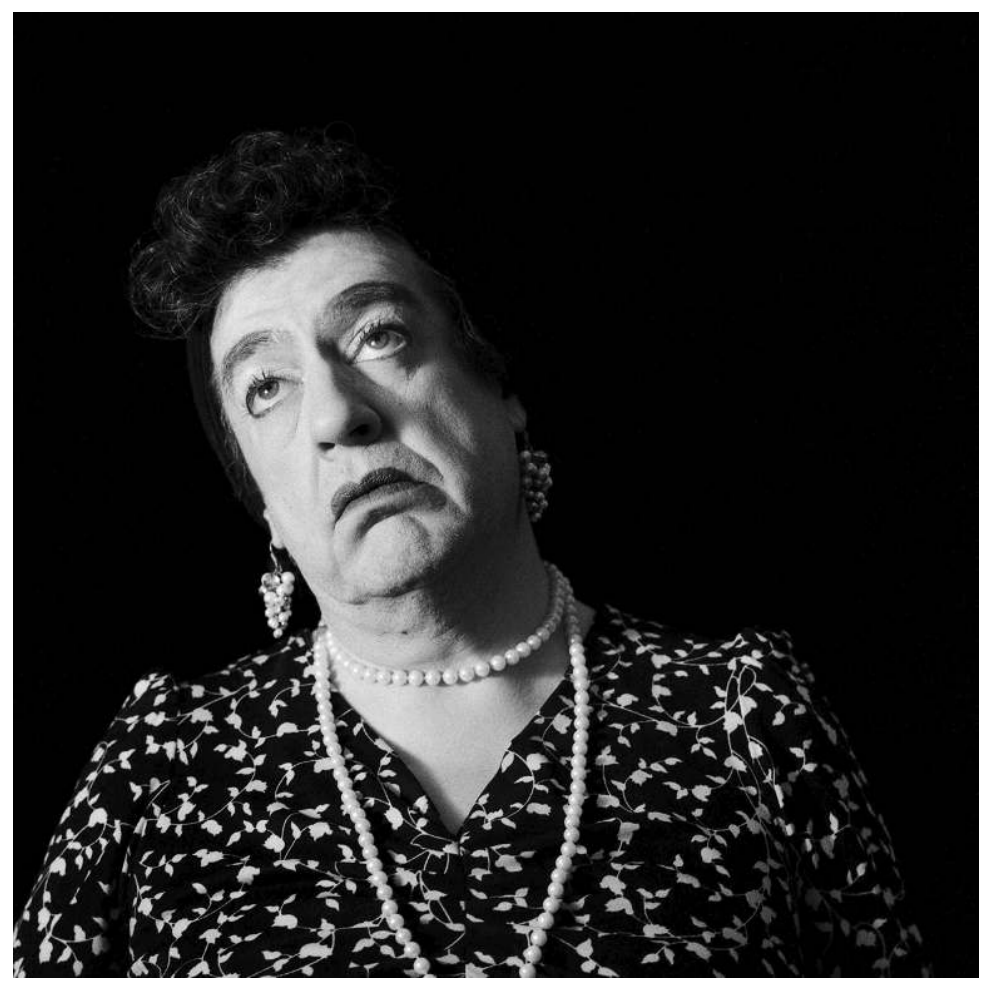

En concentrant ma série de portraits sur le seul visage de Mme Raymonde, je souligne la construction du personnage qui est devenu au fil du temps la condition même du récit. D’abord héroïne de trois pièces de théâtre de rue à l'occasion desquelles elle se faisait enlever par des gangsters, découvrait le front populaire de 36 ou fuyait la guerre, elle s'émancipe des histoires qui jalonnent sa vie et se concentre sur la pratique de son métier désormais installé de chanteuse réaliste. C'est le personnage lui-même qui sert de trame au texte et relie les chansons. Cela est du moins mon sentiment de plasticienne portraitiste à l'origine de cette série.

Le parti pris de centrer l'intrigue sur la nature même du personnage met en exergue l'art du comédien dont le corps et l'œuvre ne font qu'un. Mme Raymonde articule textes écrits, pensées et improvisations réagissant d'instinct au comportement du public, un instinct travaillé par quinze années de pratique du théâtre de rue en début de carrière où le comédien est en contact direct avec le public devenant ainsi un spécialiste de l'improvisation. Cette posture génère un rapport à soi spécifique puisque « le travail de l'acteur nécessite une triple distinction entre la personne privée (le comédien), le personnage et l'acteur sur le plateau (véhicule ou agent du personnage). Ces trois dimen- 
sions (celui qui est, celui qui joue, celui qui fait) ont tendance à se fondre en une seule au moment de l'exécution de la prouesse technique ${ }^{4} \gg$. Et c'est ainsi qu'il peut instaurer un jeu plastique avec son corps comme vecteur d'apparences et d'émotions comme le représente ma série photographique. Rompu à l'art du théâtre de rue et à l'improvisation qui marquent ses premiers pas, Denis D’Arcangelo installe désormais une proximité avec le public allant parfois jusqu'à descendre dans la salle ou s'interrompant pour s'adresser à un spectateur. Mais au-delà de sa technique de jeu, considérons aussi et dans la même logique, l'effet de son costume. La principale robe de Mme Raymonde n'est pas un costume traditionnel de théâtre. Elle n'a pas été conçue par un costumier pour la scène. Cette robe est un vêtement de prêt-à-porter, une tenue du quotidien qui souligne ainsi le réalisme du personnage.

À la fin des années quatre-vingt-dix, l'acteur, et le co-créateur de Mme Raymonde Philippe Bilheur, achètent une petite robe trouvée dans une friperie à Paris. Véritable vêtement ayant déjà vécu, cette robe est toujours le costume principal du personnage à quelques rares exceptions. Pas taillée pour un homme, la petite robe noire recouverte de feuilles blanches, s'avère plus courte que prévu, au-dessus des genoux, et étriquée aux épaules. Cette mise en scène vestimentaire joue d'un effet de maladresse renforcé par l'étonnant contraste entre les avant-bras nus du comédien semblant alors démesurés et ses bas noirs d'hiver, vraisemblablement trop épais, et chauds, pour être mis avec une robe d'été. La petite robe est sans âge, hors mode et donc indémodable, familière à toutes les générations. Elle est aussi une tenue quotidienne alors même que Mme Raymonde donne des tours de chant. La dimension quotidienne accentue la volonté du personnage de rester proche de son public rappelant ainsi que « la vie affective est $[\ldots]$ à la fois imaginaire et pratique $^{5} \gg$. Ce constat se prolonge dans l'idée développée par Morin que « les identifications imaginaires sont elles-mêmes ferments d'identifications pratiques ou mimétismes ${ }^{6} \gg$. Le public peut ainsi s'approprier le personnage, s'en sentir proche, alors que les tenues de scène plus flamboyantes transforment leurs porteurs en icônes. Mme Raymonde ironise souvent sur son statut de Diva alors qu'elle évoque, par sa forme, une chanteuse de rue de l'Entre-deuxguerres. Dans Mme Raymonde, mes plus grands succès ${ }^{7}$, elle entre en scène affublée d'un couvre-chef avec plume et paillettes digne des revues du Lido tout en portant sa petite robe habituelle. À l'aise dans sa tenue anachronique, elle prend de haut son public en lui expliquant que s'il ne l'a pas vue depuis longtemps, c'est qu'elle était prise par sa grande tournée internationale des banlieues parisiennes. L'auto-dérision de cette entrée en scène est une cri- 
tique de l'icônisation des images de la femme dans les arts du spectacle. Car rappelons, même si l'écart tend à se réduire, cette remarque de Morin : « La "mythification" s'effectue avant tout sur les stars féminines : ce sont les plus fabriquées, les plus idéalisées, les moins réelles, les plus adorées. La femme est un sujet et un objet plus mythique que l'homme, dans les conditions sociales actuelles ${ }^{8} . \gg$

D’ailleurs, le répertoire de Denis d'Arcangelo offre une place privilégiée à des chanteuses réalistes de l'Entre-deux-guerres depuis longtemps oubliées du grand public. Il protège la mémoire et rend justice à ces femmes qui ont défendu leur art en dehors de la logique actuelle du Star System qui a connu, pour les chanteurs, un renforcement non négligeable depuis les années soixante-dix avec l'avènement des vidéos clip, des captations de concerts, des diffusions télévisuelles, des réseaux sociaux, etc. Il se réfère particulièrement à la chanteuse Gaby Montbreuse. Ses références approfondies à cette artiste deviennent symptomatiques de sa posture vis-à-vis des femmes de scène en faisant d'elle un exemple révélateur. La référence récurrente à Montbreuse, faite sur le ton de l'humour, confronte la gloire passée de cette vedette des années 1910 et 1920 à la perplexité d'un public d'aujourd'hui peu réactif à l'évocation de son nom. Par exemple, dans le spectacle Mme Raymonde exagère ${ }^{9}$, notre héroïne annonce la prochaine chanson, Tu m'as possédée par surprise, d'un air entendu sans citer le nom de la fameuse Gaby Montbreuse, n'hésitant pas à dire, «mais oui, je vois que vous avez deviné ${ }^{10}$ ». La surprise et la méconnaissance du public l'autorisent alors à se lancer dans une longue présentation de son sujet. Elle entonne quelques mélodies dont la célèbre Titine, de Bertal-Maubon et Léo Daniderff, dont Montbreuse fut la créatrice en 1917 et qui inspira notamment Charlie Chaplin et Jacques Brel. Le public réagit enfin à l'écoute de cet exemple familier. À son tour perplexe, Mme Raymonde lui déclare : « ainsi vous connaissez Je cherche après Titine, oui répond le public, bon, mais vous ne connaissiez pas Gaby Montbreuse, non rétorque-t-il, bon, eh bien c'est que je vous l'ai demandé à l'envers, reprenons ${ }^{11} \ldots$.. Elle souligne ainsi le sort et le rôle des chansons populaires qui s'insinuent dans la mémoire collective tout en prenant note du fait que la postérité a oublié la créatrice d'une chanson devenue célèbre entre les mains d'interprètes masculins même si nous ne pouvons tirer aucune conclusion de ce seul exemple ${ }^{12}$. 


\section{Denis/Raymonde : portraits en pied d'une métamorphose de genre}

Denis/Raymonde est une série de quatre photographies $6 \times 6$ en noir et blanc. Les portraits sont en pied, le format carré libère ainsi un important espace noir autour du modèle pour lui donner plus d'espace et éviter l'effet colonne plus autoritaire du format rectangulaire qui installe une trop grande distance avec le spectateur. Dans la première photo, Denis D’Arcangelo apparaît en homme, pour lui-même, dans une pose simple et frontale. Proche de la neutralité, son visage est doux et fixe l'objectif dans l'attente bienveillante de la prise de vue. Dans la deuxième photo, il apparaît en sous-vêtements d'homme, en peignoir. La pose du corps reste la même, les mains dans le dos, mais le visage se fait plus dur, le regard reste fixé sur le spectateur. Connaissant mon modèle, et sa pudeur, je le mets en danger, en état de bascule. Celle-ci intervient dans la troisième photo, où il se tient cette fois en sous-vêtements féminins. La pose du corps ne change pas, le regard reste frontal et dur, mais plus fier semble-t-il comme pour défier le regard du spectateur voyeur malgré lui. Dans la dernière photo, il apparaît sous la forme définitive de Mme Raymonde. Il devient soudain expressif. Incarnant le personnage, Denis D'Arcangelo laisse place à l'interprétation dont il n'avait pas l'usage dans les photos précédentes qui le représentent en tant qu'individu, personne privée. La pose a complètement changé. Les mains sont passées devant le ventre pour tenir l'éternel sac à main du personnage; le regard sort désormais du cadre, et le visage souriant rayonne. Paradoxalement, c'est à ce moment, malgré la perte du contact visuel avec le modèle, que le contact émotionnel se délie, s'autorise, s'installe.

Cette série montre la métamorphose en quatre étapes du comédien en personnage. Les émotions sur le visage que, fait rare, je n'ai pas dirigées, me permettent d'expérimenter et d'asseoir, cette remarque de Wilde citée en introduction qui veut que le masque offre la liberté de s'exprimer. En effet, avec les modèles comédiens, je prends souvent le parti de les mettre en scène dans mon protocole artistique. Ils me comparent, d'ailleurs surpris de ce constat, plus volontiers à un metteur en scène de théâtre qu'à un photographe. Je dois alors préciser que je ne suis pas photographe, mais plasticienne de formation et que, dans mon approche, je travaille la photographie performative (au sens plasticien, mais consciente du jeu de mots avec la performance théâtrale). En faisant poser Denis D'Arcangelo, j'ai vite compris qu'il serait plus intéressant de changer mes habitudes et de limiter mes demandes pour observer son comportement spontané. Cette série met en avant cette posture puisque les 
trois premières photos sont certes posées au sens technique du terme (dans le cadre et la lumière), elles ne le sont pas au niveau expressif. Nous observons alors le passage du naturel (première photo), à la gêne (postures défensives des deux photos suivantes), pour finir avec l'incarnation d'un personnage expressif.

Rappelons ici, qu'après douze ans de théâtre de rue, Denis D'Arcangelo a : eu envie d'utiliser le personnage [...] pour un tour de chant, c'est-à-dire pour incarner une autre facette de ma personnalité. J'avais envie de faire du tour de chant et je pense que je n'aurais jamais osé le faire sous le nom de Denis D'Arcangelo. En revanche, ce personnage de Madame Raymonde possédait déjà une dimension théâtrale. Au fil des années, il était devenu mon clown, une sorte de faux double, de bouche experte pouvant dire les choses mieux que moi et de façon plus drôle et plus intéressante. J'ai donc fait mon tour de chant rêvé grâce à ce personnage $e^{13}$.

Si une certaine timidité transparaît dans cette posture, celle que nous retrouvons dans les photos, il ne peut être anodin pour un homme de vouloir interpréter pendant plusieurs décennies un personnage de femme et d'en faire le véhicule de ses désirs artistiques.
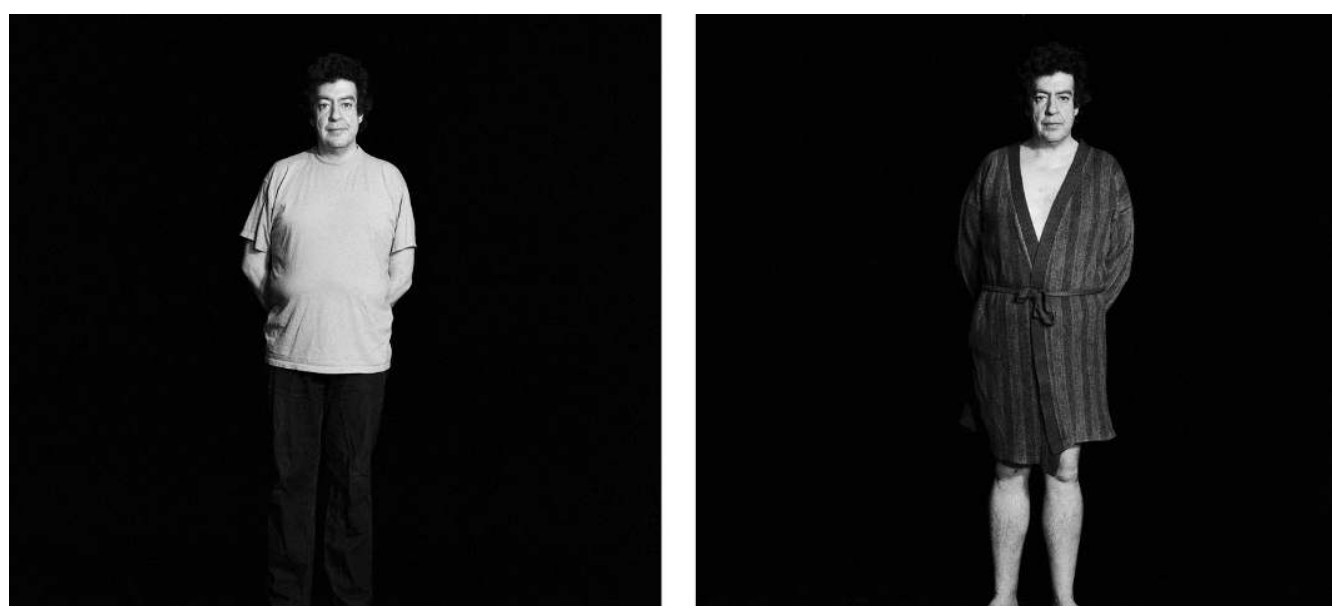
Homosexuel et féministe, le comédien peut ainsi se jouer de son personnage pour défier le regard masculin hétérosexuel (malgré tout bienvenu dans les spectacles à commencer par son accordéoniste). Cette femme, Mme Raymonde, s'incarne à travers un corps d'homme. Denis D'Arcangelo précise qu' $i$ arrive que certains spectateurs, bien qu' ils ne soient pas dupes, semblent surpris lorsqu'ils le découvrent sans fard, sous sa forme virile et pourtant reconnaissable. Ces spectateurs avaient si bien joué leur rôle en acceptant les conventions théâtrales, que Mme Raymonde avait pris corps et consistance effaçant la nature sexuelle biologique de son interprète. Ce fait est difficile à croire tant que l'on n'en a pas fait l'expérience, mais il existe deux catégories de spectateurs dans ce cas. La première concerne ceux qui ont l'information abstraite, le nom du comédien sur l'affiche, mais sont emportés par la crédibilité du personnage au point de remettre en question cette information. La deuxième concerne les curieux de passage qui n'ont pas cette information. Il arrive que les spectacles soient donnés dans des lieux ouverts, les festivals par exemple, où les personnes n'ont pas spécifiquement acheté un billet pour le voir et le découvre un peu par hasard. Il arrive aussi que des spectateurs viennent grâce à des invitations sans prendre le temps de bien se renseigner sur le spectacle... dans ces différents cas, leur découverte de l'identité du comédien peut être une vraie surprise.
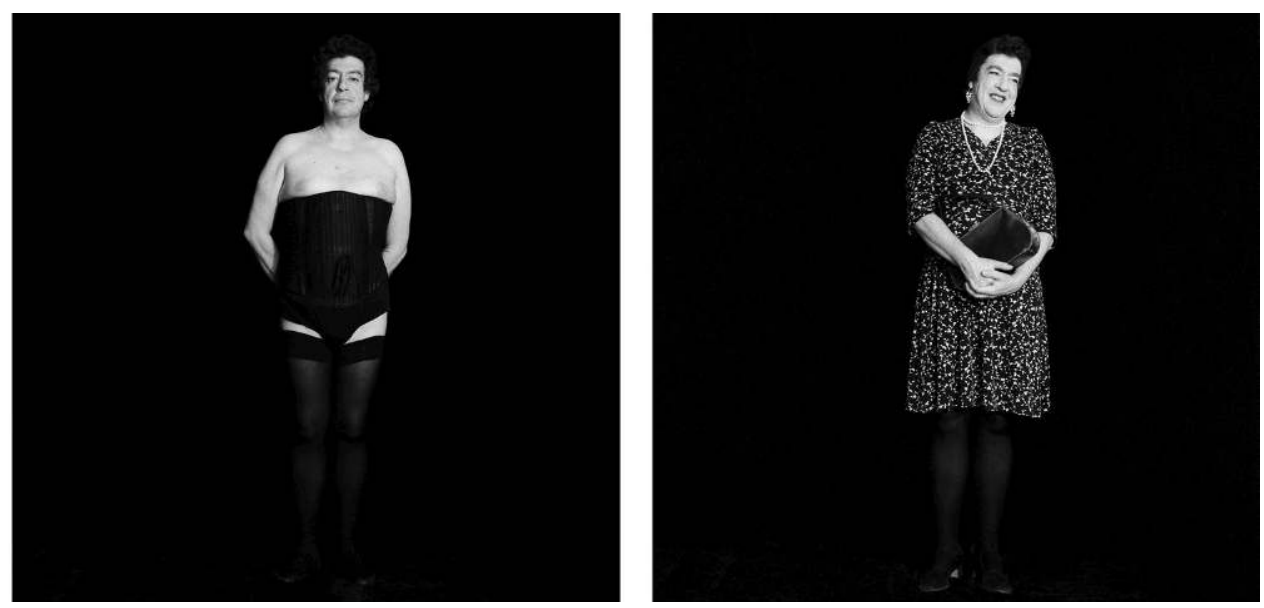
Denis D’Arcangelo rappelle ainsi que nous rejouons tous au quotidien, sans y prêter attention, les performances de genre par nos choix vestimentaires et gestuels. Les codes genrés sont si lourdement ancrés et assimilés dès la naissance que nous les acceptons comme état de nature oubliant qu'ils sont une mise en scène transmise par notre éducation. Le théâtre permet une distanciation critique. Comme l'observe Badiou :

[... l'acteur exhibe sur scène l'évaporation de toute essence stable. La fermeté des signes corporels et vocaux dont il se pare sert avant tout à établir [...] que rien ne coïncide avec soi-même. [...] En particulier, l'acteur opère contre toute théorie naturelle des différences, et en particulier de la différence des sexes. Il artificialise ce que nous croyons le plus évidemment donné, conjoint ce que nous imaginons depuis toujours séparé, sépare ce dont l'unité semblait acquise ${ }^{14}$.

Et c'est ainsi que le comédien comme travesti accomplit ce que les femmes ne pouvaient faire en recevant le regard masculin pris au piège de ses propres attentes qui construisent tant les rapports de pouvoir que de séduction. Ainsi, le choix d'interpréter un personnage de chanteuse plutôt que de chanteur permet à Denis D’Arcangelo de proposer une posture plus signifiante et plus forte. Transcendant le jeu des références et des reprises, il installe avec humour une complicité avec les femmes. «Or, comme le précise Anna Guilló parlant de l'artiste travesti, nous serions tentés de dire que c'est bien dans cette fiction du moi qu'apparaît le véritable sujet, un sujet affirmé et confirmé par le déguisement ${ }^{15}$. $>$ Mes photos soulignent ainsi la façon dont mon modèle interroge l'identité et comment, par le travestissement de genre, il traverse différents espaces à la fois physiques, mentaux, conscients, inconscients, sociaux, culturels, politiques.

Pour Denis D’Arcangelo, en tant que comédien, le corps est la condition créatrice dont l'apparence est essentielle. Il forme un discours énonçant les intentions de création qui consistent à créer une frontière poreuse entre le personnage et son créateur, le second utilisant le premier pour véhiculer un discours que les chansons, soigneusement choisies, construisent. Cette porosité installe et rappelle la métaphore de la vie comparée au théâtre qui contribue à associer de plus en plus étroitement comédien et personnage prêtant au premier les idées du second. Si bien que « le réel est baigné, côtoyé, traversé, emporté par l'irréel. L'irréel est moulé, déterminé, rationalisé, intériorisé par le réel ${ }^{16} \gg$.

Les spectacles de Mme Raymonde n'ont pas de vocation politique directe et revendiquée dans leur écriture même. Denis D’Arcangelo raconte avoir 
parfois, dans les premiers tours de chant, revendiqué les postures politiques du personnage. Il s'en est vite détourné jugeant ce choix sans intérêt artistique. De plus, « cela génère du partisanisme et ce n'est pas ce que je souhaite. À une époque, en chantant Dans un taxi de Gaby Montbreuse, j'encourageais les gens à jouer d'un instrument fictif avec la main droite qui tape et la gauche qui pense. Cela faisait rire, mais au risque de mettre mal à l'aise certains spectateurs. C'était un peu bêbête, de la simplification ${ }^{17} \gg$. Il a alors distingué sens et opinion partisane, art et militantisme.

N'oublions pas que si le personnage est imprégné par la personnalité du comédien, il ne s'agit que d'une première étape de sa construction. Ici « l'artiste travesti se (donne) en avant-première d'un travestissement du corps social, politique, artistique, médiatique ${ }^{18} \gg$. Le travestissement gagne de nouvelles strates.

\section{Le Portrait affleurant de Mme Raymonde}

Pour Le portrait affleurant de Mme Raymonde, j'ai demandé à Denis D'Arcangelo de me donner les noms de ses principales références, celles qu'il considère comme constitutives du personnage. La liste fut plus courte que je ne le pensais et hétérogène. Il m’a donné cinq noms : Arletty, Mme Raymonde, Gaby Montbreuse, Pierre Mac Orlan et Tonton Joseph (grand-oncle de l'artiste qui pratiquait lui-même, en amateur, le théâtre de rue dans des rôles de femmes). J'ai alors créé cinq portraits en diptyques. Sur chaque première photo, nous voyons la main gauche de Denis sur laquelle j'écris le nom de chaque référence. Ces mains sont des post-it, des claps de cinéma, des inscriptions qui, affleurant à même la peau du modèle, en revendiquent la nature profonde, l'identité ressentie. Sur les secondes photos de chaque diptyque, je montre le visage de Denis dans des postures qui incarnent les références citées. Nous voyons que son système de référence cumule une actrice fétiche dont il fut l'ami, un personnage de fiction, une chanteuse presque oubliée, un auteur et un membre de la famille.

Comme nous l'avons vu, bien qu'appartenant au monde du théâtre, Mme Raymonde vient d'une référence cinématographique. Denis D’Arcangelo interpelle l'univers du cinéma d'Entre-deux-guerres, qu'il associe volontiers à la chanson réaliste et aux figures féminines, qu'il s'agisse des personnages des chansons ou de leurs interprètes. Il attire ainsi l'attention sur les 
figures de femmes fortes ou du moins confrontées aux dures réalités quotidiennes des milieux populaires dans lesquels elles évoluent. Sur l'ensemble de ses spectacles, il privilégie des figures de femmes volontaires et gouailleuses comme Arletty, Gaby Montbreuse donc ou Berthe Sylva et Fréhel. En interpellant ces figures féminines familières, il rappelle que « dans nos traditions des mythologies de la théophanie et de l'incarnation, pour lesquelles il n'est de corps que par le verbe, tout système symbolique propose une fiction conductrice $^{19} \gg$. Le corps du comédien et chanteur devient un support de lecture, une représentation, seul moyen pour lui d'être lu, perçu et admis au sein d'une structure artistique et, par extension, sociale.
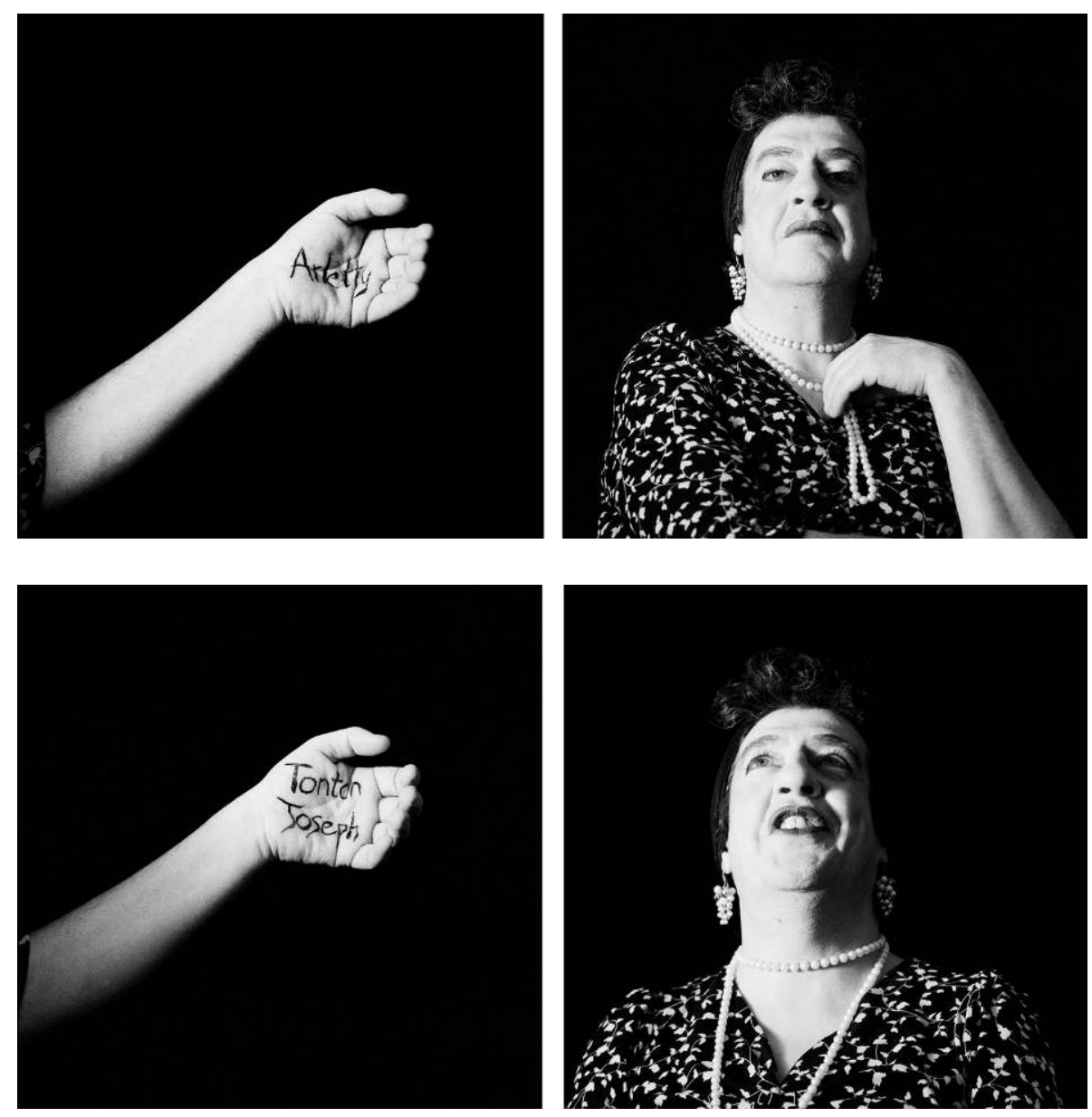
Cette série photographique représente une Mme Raymonde qui, non seulement, énonce la nature de son héritage artistique, mais qui délimite également les principaux traits de son identité par identification à ces références. Elle se joue ainsi du fameux phénomène de projection-identification, concept sociologique défini par Edgar Morin, qui désigne les liens créés entre une personne et des figures célèbres réelles ou fictives :

Toute participation affective est un complexe de projections et d'identifications. Chacun, dans la vie, soit spontanément, soit sur les suggestions d'indices ou de signes, transfère sur autrui des sentiments et des idées qu'il attribue naïvement à cet autrui. Ces processus de projection sont étroitement associés à des processus qui nous identifient plus ou moins fortement, plus ou moins spontanément à autrui. Ces phénomènes de projection-identification sont excités par tout spectacle: une action entraine d'autant plus librement notre participation psychique que nous sommes spectateurs, c'est-à-dire passifs physiquement. Nous vivons le spectacle d'une façon quasi mystique en nous intégrant mentalement aux personnages et à l'action (projection) et en les intégrant mentalement à nous (identifi(ation) ${ }^{20}$.

Ce processus permet de comprendre pourquoi nous nous sentons proches ou non d'une figure de vedette ou d'un personnage de fiction ou même de l'association des deux comme Mme Raymonde/Arletty. Nous allons désormais bien plus loin que la simple empathie sollicitée dans la première série photographique.

La projection-identification peut aussi être à l'origine d'une création. La référence est alors mise en scène. Un phénomène démultiplié dans le cas de Mme Raymonde qui transcende largement la référence initiale à Hôtel du Nord. Par cette quête identitaire nourrie de figures fictives ou idéalisées, nous observons, comme l'explique Morin, que « la projection-identification nous permet de ramener les choses figées et les essences conceptuelles à leurs processus humains ${ }^{21} \gg$. Il précise que «les projections-identifications qui caractérisent la personnalité $[\ldots]$ tendent à approcher l'imaginaire et le réel et cherchent à les nourrir l'un de l'autre ${ }^{22} \gg$. Nous observons ici une passerelle entre l'imaginaire des narrations des chansons choisies par Denis D'Arcangelo et l'existence des interprètes d'origine dont nous sont rappelés l'impact et le rôle dans le monde du spectacle. Ces interprètes sont des figures spectaculaires, accessibles essentiellement par voies médiatiques et donc mises en scène. Déjà pluridisciplinaires, les spectacles de Denis D’Arcangelo se nourrissent ainsi d'un phénomène d'intertextualité et des trouvailles glanées par 
le comédien au gré des expériences et des rencontres. Voyageant d'un réseau de codes à un autre, sa nature fictive se nourrit d'autres fictions et dévoile l'un des aspects les plus intéressants de la démarche, car le plus critique, du comédien auteur qui joue avec la notion d'original.

Ce point est renforcé par l'inversion de genre opérée dans la création de Mme Raymonde. Remarquons que Judith Butler, traitant de l'exemple du travestissement de genre dans les arts du spectacle, soutient l'idée du genre comme parodie, mais « ne présuppose pas l'existence d'un original qui serait imité par de telles identités parodiques. Au fond, la parodie porte sur l'idée même d'original ${ }^{23} \gg$. L'ambiguïté de la posture du comédien est de se référer à des figures sans original. Elles existent certes, mais leur réalité est construite et donc fictive. N'oublions pas qu'Arletty est le double spectaculaire de Léonie Bathiat.

En tant qu'artiste, et tout particulièrement avec cette série affleurante ${ }^{24}$, je travaille ce phénomène d'intertextualité qui regroupe et structure différents médiums (photographie, performance, dessin/écriture sur la peau) et différents arts (arts plastiques, arts du spectacle) stratifiant les niveaux de lecture et de perception de l'œuvre. Comme je le précisais en introduction, je ne suis pas photographe, mais plasticienne. Mes photographies cumulent volontiers les arts, comme le dessin, l'écriture et les différentes formes de performances, celles que j'assure moi-même en tant que plasticienne et celles que j'emprunte aux arts du spectacle. Je qualifie mes photographies de performatives pour souligner à la fois le cumul des pratiques, leur ouverture aux collaborations avec d'autres artistes issus d'autres arts et je rappelle surtout leur valeur d'images construites, mises en scène.

Par mes créations dérivées, j’ai voulu enrichir la démarche déjà complexe du personnage de Denis D’Arcangelo, Mme Raymonde. Ce duo devient ainsi sujet d'une création plasticienne, nouvelle strate artistique à la fois pour moi et mon modèle. 


\section{Notes}

1. Née Julia Hérissé, Gaby Montbreuse (1895-1943) est une chanteuse, artiste de musichall et actrice française. On la retrouve dans les années 1910 et 1920 aux Folies-Belleville, à l'Européen, au Château d'Eau, à la Gaîté-Montparnasse et surtout Chez Fischer jusqu'en 1927. Elle ouvre alors le Château Montbreuse tout en apparaissant dans les programmes du Concert Mayol, de la Cigale, du Casino Saint-Martin, etc. Son établissement ferme dans les années trente et elle disparaît de la scène pour se retirer en province où les conditions de la fin de sa vie restent floues.

2. Oscar Wilde, «La critique est un art - Deuxième partie », dans Intentions, Paris, le livre de poche, « classiques de poche », 2000, p. 187-188.

3. Cette confusion vient de la méconnaissance de la posture identitaire des Drags qui leur donne une valeur propre. Les transformistes et Drag Queens sont des travestis de genre dont la particularité est de projeter leurs désirs transvestiques dans une démarche esthétique que certains rendent spectaculaire et qui devient une spécialité présente dans les cabarets et les boîtes de nuit. Les enjeux qu'ils soulèvent sont très différents de ceux d'un comédien de théâtre capable d'interpréter tous les personnages. Il n'est pas justifié d'entrer dans le détail ici, mais je renvoie le lecteur à mes publications précédentes. J'y présente notamment les éléments psychologiques définis par Robert Stoller :

- Cf. Lorraine Alexandre :

- «Les Transparences ", dans Glad!, revue sur le langage, le genre, les sexualités, revue en ligne, Julie Abbou (dir.), juin 2017.

- « L'Hommage à Marcel Mariën pour commencer... : Traverser les genres », dans PlastirPlasticités, Sciences, Arts, revue en ligne, Marc-Williams Debono (dir.), juin 2016.

- «Mylène Farmer et Brigitte Bardot chez les travestis de genre : enjeux d'une référentialité ", p. 59-70, dans Chanson et performance. Mise en scène du corps dans la chanson française et francophone, Barbara Lebrun (dir.), L'Harmattan, Paris, 2012.

- «La fabrique du récit genré et sa re-signification : approche artistique », p. 39-52, dans Le Lieu du genre - La narration comme espace performatif du genre, Patrick Farges, Cécile Chamayou-Khun et Perin Emel Yavuz (dir.), Presses de la Sorbonne nouvelle, Paris, 2011.

- «La question du genre vu à travers le prisme du travestissement de genre en art ", dans Le Corps et ses genres. Les dimensions corporelles des différences sexuées, Marie Buscatto et Armelle Andro (dir.), Groupe Genre à Paris 1 : publication en ligne, 2009.

- "Les tensions entre désir et répulsion du travestissement de genre en art ", dans La Voix du regard, revue littéraire sur les arts de l'image $n^{\circ} 20$ : Images Interdites, Figures Imposées, Anna Guillo (dir.), Paris, 2007.

4. Corine Pencenat, "Athlète, acteur, artiste ? ", Le Cirque au risque de l'art, Arles, Actes Sud, 2002, p. 47.

5. Edgar Morin, Les Stars, Paris, éditions du Seuil, 1972, p. 24.

6. Ibid., p. 122.

7. Spectacle de Denis D'Arcangelo et Philippe Bilheur, avec Denis D'Arcangelo et Sébastien Mesnil à l'accordéon, joué en 2010 au Vingtième Théâtre à Paris et en tournée dans toute la France, production les Concerts Parisiens et le Vingtième Théâtre.

8. Edgar Morin, Les Stars, op. cit. 
9. Spectacle de Denis D'Arcangelo et Philippe Bilheur, avec Denis D'Arcangelo et Sébastien Mesnil à l'accordéon, joué en 2009 au Vingtième Théâtre à Paris et en tournée dans toute la France, production les Concerts Parisiens et le Vingtième Théâtre.

10. Denis D'Arcangelo, entretien avec Lorraine Alexandre et Angélique Lagarde pour Kourandart, site culturel, mars 2011.

11. Ibid.

12. Denis D'Arcangelo me signale à l'occasion d'un entretien, le 19 février 2016, qu'il existe un doute pour cet exemple précis. Certains spécialistes de la chanson pensent, sans pouvoir en apporter la preuve, que Gaby Montbreuse aurait créé une autre chanson potentiellement ressemblante de Daniderff dont elle était la compagne et fidèle interprète.

13. Ibid.

14. Alain Badiou, Rhapsodie pour le théâtre, Paris, Presses universitaires de France, 2014, p. 94.

15. Anna Guilló, «Les voleurs d'images (autoportrait et travestissement dans les pratiques du "re") ", dans La Voix du regard n 18, Paris, La Voix du regard, 2005, p. 276.

16. Edgar Morin, Le Cinéma ou l'homme imaginaire, essai d'anthropologie, Paris, Les éditions de Minuit, « arguments », 1956, p. 162.

17. Entretien avec Denis D’Arcangelo le 19 février 2016 dans le cadre de la finalisation de ce texte.

18. Anna Guilló, «Les voleurs d'images (autoportrait et travestissement dans les pratiques du "re")», op. cit., p. 280.

19. Claude Reichler, "Présentation ", Claude Reichler (sous la dir. de), Le Corps et ses fictions, Paris, Les éditions de Minuit, coll. «arguments », 1983, p. 3.

20. Edgar Morin, Les Stars, op. cit., p. 105-106.

21. Edgar Morin, Le Cinéma ou l'homme imaginaire, essai d'anthropologie, op. cit., p. 219.

22. Edgar Morin, Les Stars, op. cit., p. 23.

23. Judith Butler, Trouble dans le genre - Pour un féminisme de la subversion, Paris, La Découverte, 2005, p. 261.

24. Le terme « affleurant» est souvent utilisé pour qualifier les créations dans lesquelles j'utilise le dessin ou l'écriture sur la peau. Il permet de signaler que ces marques graphiques ne sont pas pensées comme des ajouts à l'image du modèle, mais comme des signifiants se révélant par affleurement à la surface du corps. 